\title{
A Generalized Protocol for Computational Simulation of Spray Flows Including Primary Atomization
}

Lee, T.-W. ${ }^{1^{*}}$, Greenlee, B. ${ }^{2}$, Park, J.E. ${ }^{3}$, Bellerova, H. $^{4}$, and Raudensky, M. ${ }^{5}$

$1,2,3$ Mechanical and Aerospace Engineering, SEMTE, Arizona State University, Tempe, AZ, USA

${ }^{4,5}$ Heat Laboratory, Brno University of Technology, Brno, Czech Republic

*Corresponding author email: attwl@asu.edu

\begin{abstract}
Using the theoretical result for primary atomization, we have developed a computational protocol for compact and efficient simulations of spray flows. Volume-of-fluid (VOF) is used to map out the pre-atomization liquid flow field. The velocity and liquid contour from VOF is input in the "quadratic formula" to determine the initial drop size, to furnish the initial conditions for the post-atomization discrete-particle simulations (DPS). This approach has worked quite well in pressure-atomized sprays with and without swirl, and in liquid jets in cross flows, as presented in previous ILASS papers and journals. It constitutes a general simulation framework, based on fundamental and validated fluid physics of spray atomization, and leads to a very efficient and compact computational scheme to simulate spray flows in complex geometries. In this paper, we will present application examples in various other spray geometries and discuss potential uses in practical devices and systems, so that the generalizability of the method is established. This approach is easy to implement, generalizable, and thus can be used in complex injection systems by first analysing the internal fluid motion and using it to advance the scheme to atomization and post-atomization states.
\end{abstract}

\section{Keywords}

Computational, primary atomization, spray flow simulations

\section{Introduction}

Computability of spray flows is an important issue, from both fundamental and practical perspectives [1-3]. Spray flows have important applications in fuel injection, agriculture, medical devices, and industrial processes such as spray cooling. For this reason, many efforts have been devoted to experimental, computational and also some theoretical aspects of spray flows. In particular, spray atomization is a central and probably the most difficult aspect of spray flows. It involves deformation of the liquid opposed by surface tension and viscous forces, often leading to complex and very small liquid interfacial features. Surface tension is a curvature-dependent effect, the length scale of which often descends below the Kolmogorov scale in high-speed spray flows, thus extending the spatial and temporal resolution requirements during computation. For this reason, direct numerical simulations can exhibit realistic liquid deformation and interface features down to fairly small scales, but are highly strained for resolution and accuracy when simulating the final drop formation at high Reynolds and Weber numbers of practical importance. Large eddy simulations and turbulence models based on Reynolds-averaged Navier-Stokes equations extenuate the computational requirements, but then require primary atomization models since the length scales of the drop formation are below the spatial resolution. Several such primary atomization models have been devised and implemented [4-8]. Once the primary drop size has been produced in this manner, then that data can be used as the initial size and velocity for the subsequent "dispersed-phase" simulations, which often is a Eulerian-Lagrangian type of algorithm to track 
the droplet motion while allowing for momentum exchange with the surrounding gas medium. For evaporation and combustion, inter-phase mass and energy transport can also be added. Thus, the computational procedure for spray flows can be considered as a combination of continuous liquid phase, primary atomization, and then dispersed-phase simulations. Secondary atomization or coalescence effects can be added if conditions render them significant. Here, we assess that the large-eddy or turbulence models are sufficiently accurate for many applications for the continuous liquid phase part of the simulations [8, 9], for moderate length scales safely above the atomization scales. Also, the Lagrangian type of droplet tracking is deemed accurate, where current droplet motion and turbulent dispersion models found in commercially available computational software (e.g. ANSYS-Fluent) exhibit realistic droplet trajectories $[10,11]$. Therefore, the key requirement for a robust computational procedure for spray flows is the module for the primary atomization. If this component is in place, then coarse-grid simulations of spray flows are possible to generate accurate spray flow simulations. In this regard, we have developed an analytical framework for spray atomization using the integral form of the conservation equations for various spray geometries: pressureatomized sprays with and without swirl [12-15], liquid injection into cross-flows [16], liquid injection with co-flow of air, otherwise known as air-blast atomization [17], and secondary atomization [14]. By using the integral form, we are able to by-pass the complexity of the fluid physics, and relate the initial mass, energy and momentum flow rates to the final state. This process leads to an explicit expression for the drop size which has been extensively validated [12-15].

\section{Computational Procedure}

As noted above, we combine the established simulation methods for the continuous liquid phase prior to atomization, and dispersed phase after that. In between, we insert either Equation 1 or 2 as the primary atomization module, depending on the spray geometry. For pressure-atomized liquid jets with or without swirl, the conservation laws of mass and energy lead to the $D_{32}$ expression below [12-15]:

$$
D_{32}=\frac{3 \sigma+\sqrt{9 \sigma^{2}+K^{\prime} \frac{\rho_{L} \mu \bar{u}^{2}}{u_{i n j}} \frac{u_{i n j}^{2}-\bar{u}^{2}}{2}}}{\rho_{L} \frac{u_{i n j}^{2}-\bar{u}^{2}}{2}}
$$

Local liquid velocities from the continuous liquid simulation are used as input into Equation 1, for determination of the drop size. For gas-driven atomization processes, we add the gasphase kinetic energy terms at the initial and final states, leading to the following $D_{32}$ expression for liquid injection in cross-flows and air-blast atomization [16, 17].

$D_{32}=\frac{3 \sigma+\sqrt{9 \sigma^{2}+\frac{K \mu_{L}\left(u_{i n}-u_{\text {inj }}\right)^{2}}{u_{\text {inj }} A_{\text {inj }}}\left[\frac{\rho_{L} u_{\text {inj }}^{2}}{2}\left(1-\left(\frac{u_{L}}{u_{\text {inj }}}\right)^{2}\right)+\frac{u_{\text {in }} A_{\text {in }} \rho g u_{\text {in }}^{2}}{u_{\text {inj }} A_{\text {inj }}}{ }^{2}\left(1-\left(\frac{u_{\text {out }}}{u_{\text {in }}}\right)^{2}\right)\right]}}{\left[\frac{\rho_{L} u_{\text {inj }}^{2}}{2}\left(1-\left(\frac{u_{L}}{u_{\text {inj }}}\right)^{2}\right)+\frac{u_{\text {in }} A_{\text {in }} \rho g u_{\text {in }}^{2}}{u_{\text {inj }} A_{\text {inj }}}\left(1-\left(\frac{u_{\text {out }}}{u_{\text {in }}}\right)^{2}\right)\right]}$

For liquid jets in cross flow and other air-assisted atomization processes, we would opt for Equation 2, as it includes the gas kinetic energy terms. Liquid and gas velocities are input into Equation 2, to determine the local $D_{32}$.

The computational procedure can be summarized as follows: 
1. Run liquid-phase simulations, e.g. volume-of-fluid (VOF), to obtain liquid core trajectory and velocities for the liquid and gas phase.

2. Apply the atomization criterion.

3. Use the velocities from Step 1 in $D_{32}$ equation (Eq. 2) to find the local initial drop size, and also to set the local initial drop velocity.

4. Run the Lagrangian discrete particle simulations to track the droplets, using the above initial drop size and velocities.

Figure 1 shows the schematic of the above procedure, where the liquid volume fraction and velocities from the VOF simulation are used in steps 2 and 3. Then, discrete-phase model (DPM) can be run. Both the VOF and DPM are run on the ANSYS-Fluent platform for the current work. The sequence is performed in series, but can be automated using user-defined functions and macro-programming. To account for turbulence, Reynolds-averaged NavierStokes, two-equation models are utilized. Only the mean velocities are needed so that steadystate simulations are sufficient, requiring minimal computational resources.

The atomization criterion is tailored to best capture the most-probably locations of atomization for the specific spray geometry. For example, exemplified in Figure 1, in axial jets, the atomization locations are determined the maximum magnitude of the lateral and axial velocity gradients, for surface and primary (i.e. tip of the jet) atomization locations respectively. For pressure atomized sprays with swirl, the atomization criterion is applied as an incremental progression downstream of the nozzle along the liquid sheet to account for further atomization of larger liquid structures that are sheared from the sheet upstream. In the perpendicular direction (with respect to the liquid propagation direction), the maximum magnitude of the shear rate bounds each droplet-forming profile. An example of the atomization criteria for pressure atomized sprays with swirl is shown below in Figure 2.
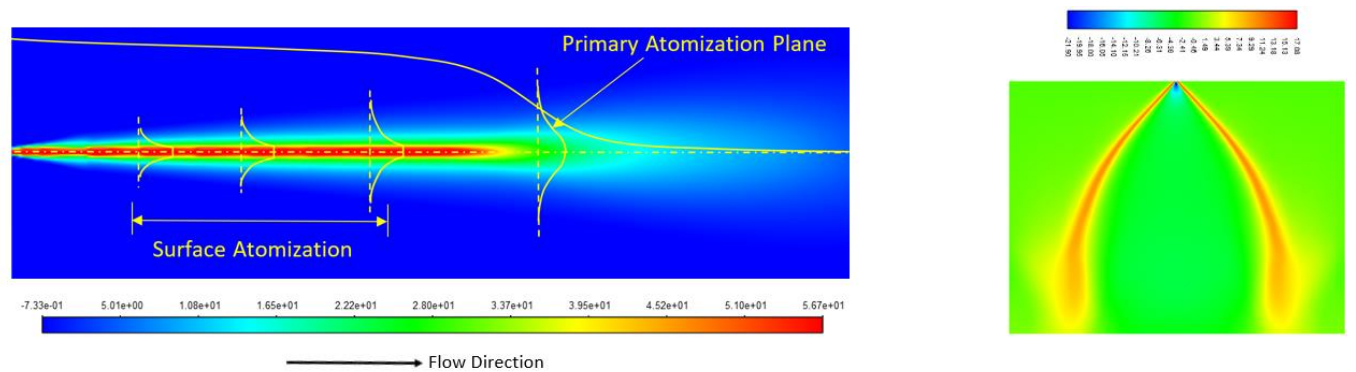

Figure 1 (left). A schematic of the computational protocol. The continuous liquid part is computed (Step 1), then local $D_{32}$ (from Eq. 3) and liquid velocity (Step 3) are used to initiate the discrete phase simulation (Step 4) at the surface and the primary atomization plane. Contour plot is for the liquid velocity in $\mathrm{m} / \mathrm{s}$.

Figure 2 (right). Liquid velocity contour map for swirl sprays in $\mathrm{m} / \mathrm{s}$. Velocity profiles are used along the liquid sheet, to initiate the discrete phase computations, as schematically shown (locations are approximate, for illustration purposes).

The atomization criterion for liquid jets in cross flow is based on momentum and energy balance analyses. This indicates that the gas motion is the primary cause for liquid atomization. Therefore, we adopt a criterion of prorated atomization on the backside of the liquid column. The reduction of gas momentum is associated with increase of liquid momentum. By sampling the gas velocities downstream of the liquid column from the VOF results, we can obtain a good estimate of the momentum state. This momentum state is used to determine the atomization rate, or the number flux of the droplets to be released, which is set to inversely related to the downstream gas velocity. I.e. the less the gas momentum defect the less the number flux. Of course, the total number flux converted to the mass flow rate 
must equal the injected mass flow rate. The gas velocity data is also input into Equation 2 to determine the local $D_{32}$. Then, the droplets of $D_{32}$ at the local gas velocity are released at equally-spaced points along the curved liquid column (shown in Figure 3), and tracked in dispersed phase computation (DPM) during Step 4.

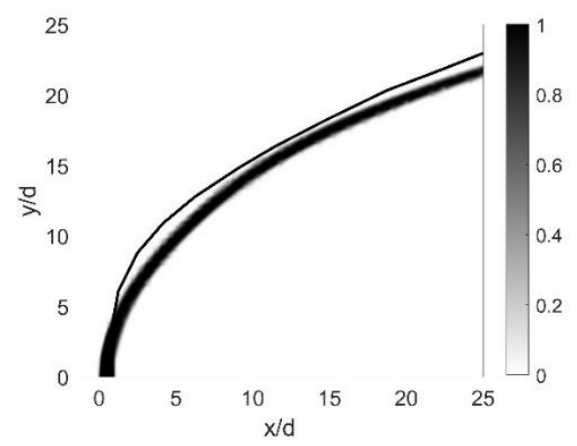

Figure 3. Computed liquid column trajectory during the VOF part of the simulation (Step 1). Experimentally observed trajectory (line) is from Mashayek (2006) [22]. The contour color bar indicates liquid volume fraction.

Upon furnishing the initial drop sizes and momentum states, the droplets are tracked as pointparticles in an Eulerian-Lagrangian algorithm, where the momentum transfer between the liquid droplets and the surrounding gas media are allowed. This provides realistic droplet trajectories. Interphase mass and energy transfer can be added as well to account for droplet heat transfer, evaporation and combustion.

In summary, we can consider this approach as using the integral form of conservation of mass and energy, to relate the drop size to the liquid and gas kinetic energy (velocities). The momentum equations are solved in the differential form, with CFD, to obtain the velocity field, so that we are making use of the full set of conservation equations in dual modes (integral and differential) to solve for all of the unknowns in spray flows. Also, in spite of relying only on the differential numerics, we are making as much use of the analytics as possible to achieve computable set of equations, at conditions applicable to practical injection systems.

\section{Results and Discussion}

We first begin by viewing the structure and number concentration (density) of the computed spray flow. Using the above sequence of computations, the general appearance of the spray flow fields displays characteristics reminiscent of experimentally observed sprays. An example for liquid jets in high-speed cross flow is provided in Figure 4. The droplets are concentrated in the mid-region of the liquid column, with slightly upward trajectories. The overall shape and appearance of the spray from the simulation is similar to experimental images, under similar momentum ratios, e.g. Becker and Hassa [18]. This gives positive support for the use of the momentum and mass analysis in specifying the released droplet mass flux for liquid jets in cross flow.

In addition to spray flow visualization, quantifiable comparisons to experimental data are performed. In particular, Sauter-mean diameter (SMD), droplet velocities, volumetric flux, impingement pressure and particle-wall heat transfer coefficient (HTC) are computed and compared with the appropriate experimental data sets. Below, we demonstrate comparisons to experimental data generated by this general computational procedure in multiple spray geometries. 

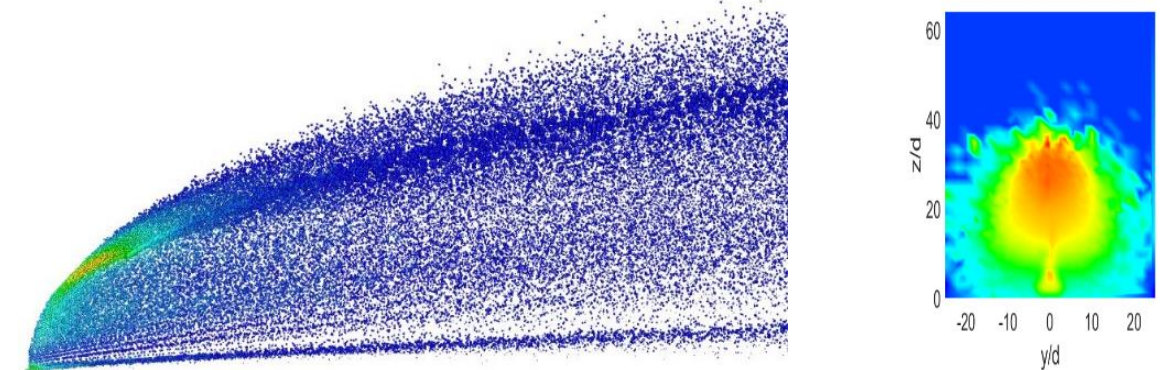

Figure 4. An example of the spray flow simulation using the current computational protocol, including the primary atomization, for liquid jets in high-speed cross flow. Particles colored by number density. Side (left) and frontal (right) views.
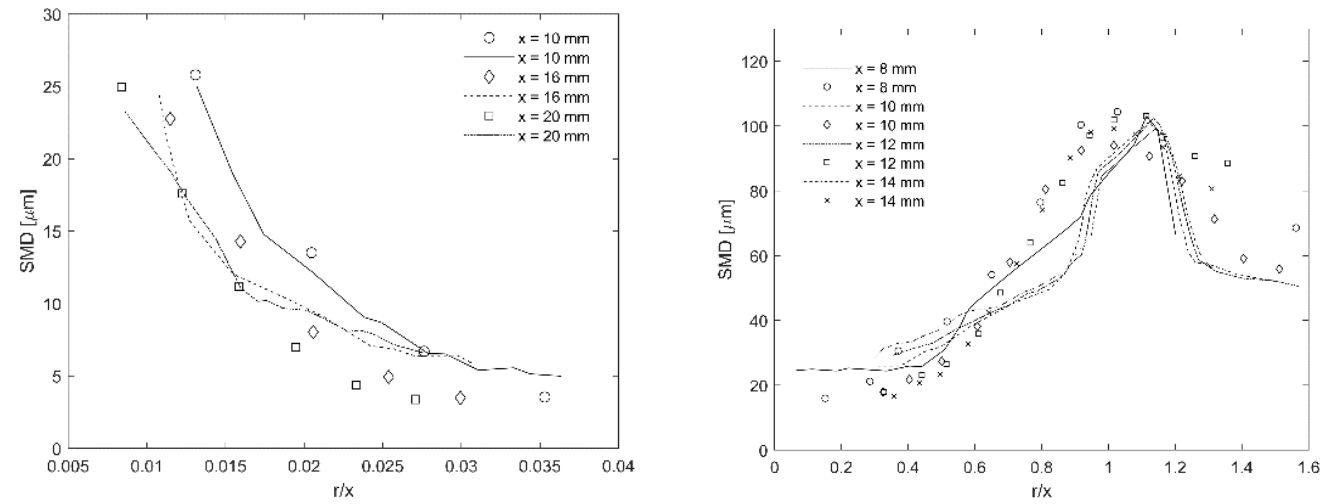

Figure 5a (left). Sauter-mean diameter comparison of a diesel axial injection between computational and experimental results [19]; 5b (right). Sauter-mean diameter comparison of a kerosene injection with swirl between computational and experimental results [20].

Through these comparisons, we validate our computational procedure. As demonstrated in Figures $5 \mathrm{a}$ and $5 \mathrm{~b}$, the particle sizes are captured with decent agreement with experimental data. Considering the fact that there are inherent uncertainties in measurements of drop size and mass flux, and also that even advanced CFD methods do not generate convincing drop size results without an established primary atomization model, quantitatively viable results point toward viability of the current protocol.

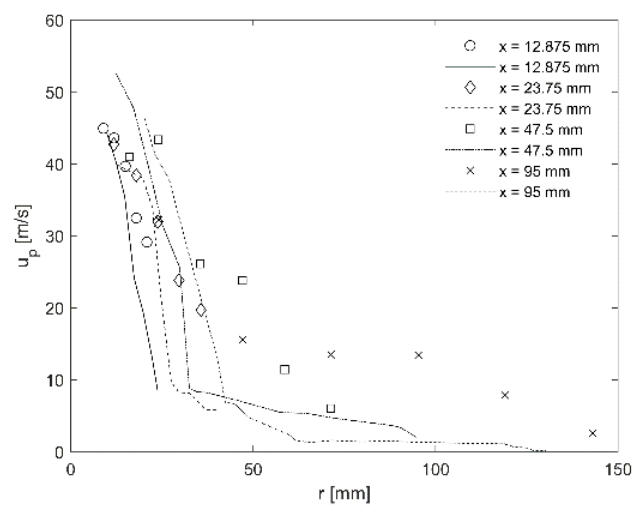

Figure 6. Normalized particle velocity comparison of a large nozzle water axial injection between computational and experimental results [21].

The computed particle velocities are similarly compared with data (Figures 6 and $7 \mathrm{a}$ ), with some underestimates attributed to momentum discrepancies in the initial energetic states of 
the particles due to additional drag forces in the VOF solutions. The liquid is assumed intact, whereas if the atomization simulated to occur in a VOF-DPS computation (focus of future works) the observed drag reduction on the liquid can be captured, leading to higher fidelity initial particle momentum.
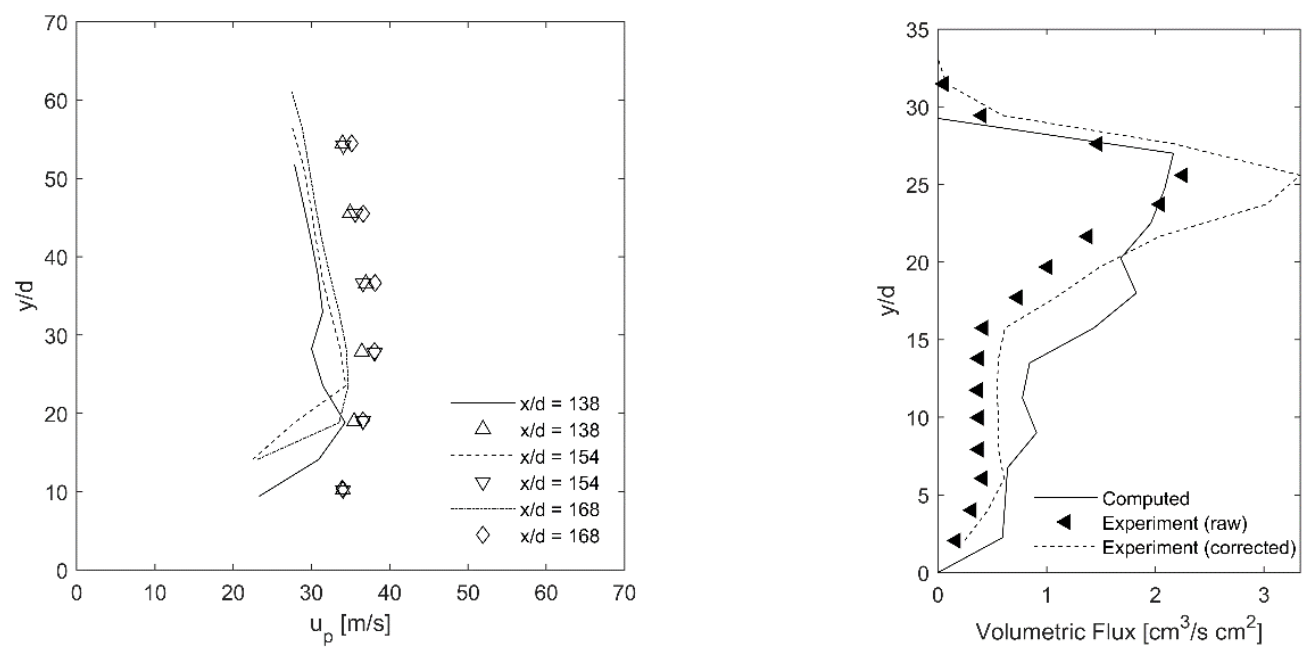

Figure 7a. Particle velocity comparison of a liquid jet in cross flow between computational (lines) and experimental (symbols) results [22]; and 7b. Particle volumetric flux comparison of a liquid jet in cross flow between computational (solid line) and experimental (symbols and broken line for corrected from signal drop-off) results [23].

Another quantity of interest is the particle volumetric flux, particularly for liquid jets in cross flow. In Figure 7b, comparison between computation and experimental volumetric flux is displayed for a liquid jet in high-speed cross flow. Experimentally, the volumetric flux is difficult to measure due to odd-shaped liquid parcels and smaller droplets hidden behind larger droplets, therefore the experimental data is corrected to account for the entire volumetric flux of the injected liquid. This means that it is very important to be able to simulate this quantity and we have shown that the general computational procedure applied is accurate is this regard and very useful for design processes (i.e. turbine combustor systems).

Another geometry that has been tested is air-assist spray flows, where drop size, velocities, and impact pressure (Figure 8) all agree with experimental data [24].

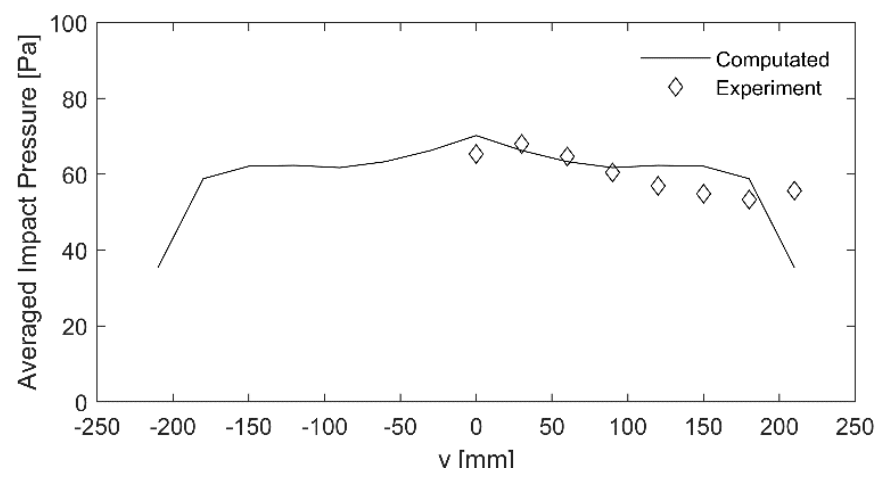

Figure 8. Particle-wall impingement pressure comparison for an air-assist flat spray between computational and experimental results [24]. 


\section{Conclusions}

From our previous work, a generalized primary atomization module, based around the integral formulation of the conservation principles, leads to the quadratic formula for determination of drop size in various spray geometries. This can be integrated with CFD for a general computational protocol for spray flows including primary atomization. The implementation comprises of four steps: (1) continuous liquid simulation; (2) application of the atomization criterion; (3) specification of the initial drop size and velocity; and (4) dispersed droplet trajectory calculations. Comparison between experimental data and the computed results show that this procedure works quite well. Use of the time-averaged VOF or similar for the continuous liquid phase simulation and Lagrangian droplet tracking for the dispersed phase requires minimal (coarse grids) computational resources, and yet generates results with good accuracy at realistic Reynolds and Weber number conditions found in practical injection systems. This protocol is easy to implement, computationally efficient, and robust in producing realistic spray flow simulations including the drop size, velocity, droplet impact pressure, particle-wall heat transfer coefficients and other relevant spray contours. In this work, we demonstrate the generalizability of this protocol.

\section{Acknowledgments}

This work was supported in part, for the Czech researchers, by the Ministry of Education (Czech Republic), under the program INTER_ EXCELLENCE (project number LTAUSA19053).

\section{Nomenclature}

$\mathrm{A}_{\text {inj }} \quad$ liquid injector exit area $\left[\mathrm{m}^{2}\right]$

$A_{\text {in }} \quad$ cross-sectional area of incoming gas flow $\left[\mathrm{m}^{2}\right]$

$A_{\text {out }}$ cross-sectional area of the outgoing gas flow (usually, $A_{\text {out }}$ is set equal to $A_{\text {in }}$ ) $\left[\mathrm{m}^{2}\right]$

$\mathrm{d}_{\mathrm{in}} \quad$ diameter of the air jet at the injector diameter $[\mathrm{m}]$

$\mathrm{d}_{\mathrm{inj}} \quad$ injector diameter $[\mathrm{m}]$

$D_{32} \quad$ Sauter mean diameter $[\mu m]$

SMD Sauter mean diameter $[\mu m]$

$\mathrm{K}, \mathrm{K}^{\prime}$ proportionality constants for the viscous dissipation term $[s]$

$u_{\text {inj }} \quad$ mean injection velocity $\left[\mathrm{ms}^{-1}\right]$

$\mathrm{u}_{\mathrm{L}} \quad$ mean drop velocity $\left[\mathrm{m} \mathrm{s}^{-1}\right]$

uout velocity of the outgoing air $\left[\mathrm{m} \mathrm{s}^{-1}\right]$

$u_{p} \quad$ particle velocity $\left[\mathrm{ms}^{-1}\right]$

$\mu_{L} \quad$ liquid viscosity [Pas]

$\rho_{g} \quad$ ambient gas density $\left[\mathrm{kg} \mathrm{m}^{-3}\right]$

$\rho_{L} \quad$ liquid density $\left[\mathrm{kg} \mathrm{m}^{-3}\right]$

$\sigma \quad$ surface tension $\left[\mathrm{N} \mathrm{m}^{-1}\right]$

HTC heat transfer coefficient $\left[W m^{-2} K^{-1}\right]$

\section{References}

[1] Strasser, W. and Battaglia, F., 2016, Identification of Pulsation Mechanism in a Transonic ThreeStream Airblast Injector, Journal of Fluids Engineering, 38(11): 111303.

[2] Strasser, W., 2008, Discrete Particle Study of Turbulence Coupling in a Confined Jet Gas-Liquid Separator, Journal of Fluids Engineering, 130(1): 011101. 
[3] Wang, Y., Zhang, F., Yuan, S, Chen, K., Wei, X, and Appiah, D., 2020, Effect of URANS and Hybrid RANS-Large Eddy Simulation Turbulence Models on Unsteady Turbulent Flows Inside a Side Channel Pump, Journal of Fluids Engineering, 142(6): 061503.

[4] Movaghar, A., Linne, M., Herrmann, M., Kerstein, A.R. and Oevermann, M., 2018, Modeling and numerical study of primary break-up under diesel conditions, International Journal of Multiphase Flows, 98, 110-119.

[5] Umemura, A. and Shinjo, J., 2018, Detailed SGS atomization model and its implementation to twophase LES, Combustion and Flame, 195, 232-252.

[6] Saeedipour, M., Pirker, S., Bozorgi, S., and Schneiderbauer, S., 2016, An Eulerian-Lagrangian hybrid model for the coarse-grid simulation of turbulent liquid jet breakup, International Journal of Multiphase Flows, 82, 17-26.

[7] Strasser, W., 2008, Discrete Particle Study of Turbulence Coupling in a Confined Jet Gas-Liquid Separator, Journal of Fluids Engineering, 130 (1), 011101.

[8] Shi, H. and Kleinstreuer, C., 2007, Simulation and Analysis of High-Speed Droplet Spray Dynamics, Journal of Fluids Engineering, 129(5), 621-633.

[9] Dhakal, T.P., Walters, D.K., and Strasser, W., 2014, Numerical study of gas-cyclone airflow: An investigation of turbulence modelling approaches, International Journal of Computational Fluid Dynamics 28(1-2), 1-15.

[10] Pougatch, K., Salcudean, M., 2011, Computational Investigation of Liquid Spray Dispersion Modification by Conical Nozzle Attachments, Journal of Fluids Engineering, 133(3): 031301.

[11] Patro, P. and Dash, S. K, 2014, Computations of Particle-Laden Turbulent Jet Flows Based on Eulerian Model, Journal of Fluids Engineering, 136(1): 011301.

[12] Lee, T.-W. and Robinson, D., A Method for Direct Calculations of the Drop Size Distribution and Velocities from the Integral Form of the Conservation Equations, Combustion Science and Technology, 183(3), pp. 271-284, 2011.

[13] Lee, T.-W. and An, K., 2016, Quadratic Formula for Determining the Drop Size in PressureAtomized Sprays with and without Swirl, Physics of Fluids, 28, 063302.

[14] Lee, T.-W. and Lee, J.Y., 2012, Momentum Effects on Drop Size, Calculated Using the Integral Form of the Conservation Equations, Combustion Science and Technology, 184, pp. 434-443.

[15] Lee, T.-W. and Ryu, J.H., 2014, Analyses of Spray Break-Up Mechanisms Using the Integral Form of the Conservation Equations, Combustion Theory and Modeling, 18(1), pp. 89-100.

[16] Lee, T.-W., Park, J.E. and Kurose, R., 2017, Determination of the drop size during atomization of liquid jets in cross flows, Atomization and Sprays, 28, 3, 241-254.

[17] Lee, T.-W. and Park, J.E., 2019, Determination of the drop size during air-blast atomization, Journal of Fluids Engineering, 141 (12), 121301.

[18] Becker, J. and Hassa, C., "Breakup and Atomization of a Kerosene Jet in Crossflow at Elevated Pressure", Atomization and Sprays, Vol. 12, pp. 49-67, 2002.

[19] Martinez, G. L., Magnotti, G.M., Knox, B.W., Genzale, G.L., Matusik, K.E., Duke, D.J, Powell, C.F., and Kastengren, A.L, Quantification of Sauter Mean Diameter in Diesel Sprays using ScatteringAbsorption Extinction Diesel, ILASS-Americas 29th Annual Conference on Liquid Atomization and Spray Systems, Atlanta, GA, May 2017.

[20] Marchione, T., Allouis, C., Amoresano, A., Beretta, Federico, 2007, Experimental

investigation of a pressure swirl atomizer spray, Journal of Propulsion and Power, doi: 10.2514/1.28513. [21] Ruff GA, Bernal LP, Faeth GM., 1991, Structure of the near-injector region of nonevaporating pressure-atomized sprays, Journal of Propulsion and Power, 7:221-230.

[22] Mashayek, A., Experimental and Numerical Study of Liquid Jets in Cross Flow, M.Sc Thesis, University of Toronto, 2006.

[23] Song, J., Cain, C.C. and Lee, J.G., Liquid jets in subsonic air crossflow at elevated pressure, Journal of Engineering for Gas-Turbines and Power, Vol. 137, No. 4, 041502, 2014.

[24] Kotrbacek, P., Bellerova, H., Luks, T. and Raudensky, M. (2021), Heat Transfer Correlations for Secondary Cooling in Continuous Casting. steel research int. 2000465. https://doi.org/10.1002/srin.202000465 